\title{
RB-PIRULÍ: VARIEDAD DE SORGO DULCE PARA PRODUCCIÓN DE BIOMASA Y AZÚCARES EN MÉXICO
}

\author{
RB-PIRULÍ: SWEET SORGHUM VARIETY FOR BIOMASS AND \\ SUGAR PRODUCTION IN MEXICO
}

\section{Noé Montes-García', Sergio Uribe-Gómez², Ma. Eugenia Cisneros-López*, Víctor Pecina-Quintero ${ }^{3}$, Tomás Moreno-Gallegos ${ }^{4}$ y Arturo Díaz-Franco ${ }^{1}$}

\begin{abstract}
'Instituto Nacional de Investigaciones Forestales Agrícolas y Pecuarias (INIFAP), Campo Experimental Río Bravo, Río, Bravo Tamaulipas, México. ${ }^{2}$ INIFAP, Campo Experimental Cotaxtla, Paso del Toro, Municipio de Medellín, Veracruz, México. ${ }^{3}$ NIFAP, Campo Experimental Bajío, Celaya Guanajuato, México. ${ }^{4}$ INIFAP, Campo Experimental Valle de Culiacán, Costa Rica, Culiacán, Sinaloa, México.
\end{abstract}

*Autor para correspondencia (cisneros.maria@inifap.gob.mx)

El sorgo dulce [Sorghum bicolor (L.) Moench] se cultiva en áreas tropicales y subtropicales del mundo y se caracteriza por sus altos contenidos de carbohidratos estructurales (lignina, celulosa y hemicelulosa) y de azúcares solubles (sacarosa, fructosa y glucosa) que pueden emplearse para producir etanol (Murray et al., 2009). En México, el sorgo dulce se ha evaluado como cultivo alternativo en Tamaulipas para el ciclo otoño-invierno (O-I) y primaveraverano (P-V) (Montes et al., 2010). En climas donde la caña de azúcar (Saccharum officinarum L.) se cultiva ampliamente, o en áreas marginales donde la caña ya no es rentable, el sorgo dulce podría ser un sistema de producción complementario.

En Estados Unidos de América, durante las décadas de los 1960's y 1970's se desarrollaron variedades de sorgo dulce en la Estación Experimental Meridian, Mississippi, a partir de accesiones de África (Murray et al., 2009). El programa de mejoramiento genético de sorgo dulce del Instituto Nacional de Investigaciones Forestales, Agrícolas y Pecuarias (INIFAP), localizado en el Campo Experimental Río Bravo y el Centro de Investigación Regional del Noreste (CERIB-CIRNE, Tamaulipas), se inició con la introducción de variedades provenientes de la Estación Meridian en el año 2006. Al sembrarlas en Río Bravo, estas introducciones presentaron susceptibilidad a plagas y enfermedades, sensibilidad al fotoperiodo, pobre calidad de semilla y bajo rendimiento de grano, floración y madurez fisiológica del grano tardía, variabilidad dentro de cada introducción y acame. La variedad RB-Cañero fue resultado de la etapa inicial de mejoramiento de sorgo dulce en México (Montes et al., 2010).

El sorgo dulce RB-Pirulí es una variedad de polinización libre que se derivó de la población experimental PSD-003 en el CERIB-CIRNE, producto de la recombinación de seis variedades de sorgo dulce (Indu, Urja, Dale, Theis, Topper 76-6 y M-81E) introducidas de Estados Unidos de América.
En el ciclo 0-I 2009 se sembraron en un lote aproximadamente 500 plantas por variedad, las cuales se recombinaron genéticamente gracias al grado de polinización cruzada que el sorgo presenta de manera natural, la cual puede ser hasta $10 \%$, según el genotipo (Barnaud et al., 2008) y las condiciones climáticas (Pedersen et al., 1998).

A la cosecha, en cada variedad se seleccionaron 60 plantas con base en su tolerancia al acame, precocidad, producción de semilla por panoja y contenido de azúcares en el tallo. Las panojas se desgranaron manualmente y se formó un compuesto balanceado de semilla de cada panoja, el cual se sembró en un lote en el ciclo P-V 2009 con 1200 plantas aproximadamente. La selección fenotípica se realizó con los mismos parámetros; al final, permanecieron 300 plantas. En el ciclo P-V 2010 se inició la selección individual mediante el método de pedigrí, en la que se eligieron 30 familias por su precocidad, uniformidad, producción de biomasa, azúcares, jugo y producción de semilla. La selección individual continuó por cuatro generaciones más hasta el ciclo 0-I de 2013. La genealogía de la variedad RB-Pirulí es 10-3-1-1.

Del ciclo P-V 2013 al ciclo P-V 2014 se llevaron a cabo evaluaciones en varias localidades del trópico de México (Cuadro 1). Durante el ciclo O-I 2015 se incrementó la semilla y en los ciclos O-I y P-V 2016 se efectuó la caracterización morfológica en Río Bravo, Tamaulipas.

RB-Pirulí produce hasta dos macollos, su floración se presenta a los $80 \mathrm{~d}$ y el primer corte puede hacerse entre los 110 y $120 \mathrm{~d}$. Cuando se aplica un segundo corte, la producción de biomasa puede reducirse según la fecha de siembra. La altura de planta en promedio es de $2.18 \mathrm{~m}$, aunque puede superar los $3 \mathrm{~m}$, el diámetro de tallo varía entre 15 y $20 \mathrm{~mm}$ y muestra tolerancia al acame (Figura 1). Es insensible al fotoperiodo y presenta buena producción 
Cuadro 1. Producción de biomasa y de azúcares de la variedad RB-Pirulí y otras variedades de sorgo dulce en diversos ambientes del trópico de México.

\begin{tabular}{|c|c|c|c|c|c|c|c|c|c|c|}
\hline \multirow{2}{*}{ Genotipo } & BTA & BTO & Jugo & ${ }^{\circ}$ Brix & ATJ & BTA & BTO & Jugo & ${ }^{\circ}$ Brix & ATJ \\
\hline & \multicolumn{2}{|c|}{$\left(\mathrm{t} \mathrm{ha}^{-1}\right)$} & $\left(\mathrm{Lha}^{-1}\right)$ & & $\left(g^{-1}\right)$ & & & $\left(\right.$ L ha $\left.^{-1}\right)$ & & $\left(g L^{-1}\right)$ \\
\hline & \multicolumn{5}{|c|}{ Tehuantepec, Oaxaca O-I 2014 (riego) } & \multicolumn{5}{|c|}{ Río Bravo, Tamaulipas O-I 2014 (riego) } \\
\hline RB-Pirulí & $22 \mathrm{a}$ & $34 \mathrm{a}$ & $6,400 \mathrm{a}$ & $17 \mathrm{a}$ & $182 \mathrm{a}$ & $49 a$ & $68 \mathrm{a}$ & $14,200 \mathrm{a}$ & 13 a & $152 \mathrm{a}$ \\
\hline RB-Cañero & $16 b$ & $25 a$ & $4,600 \mathrm{c}$ & $14 \mathrm{~b}$ & $157 b$ & $45 b$ & $58 b$ & 13,000 a & $8 b$ & $113 b$ \\
\hline G-Star 501 & $13 b$ & $21 b$ & $3,800 \mathrm{~d}$ & $12 b$ & $126 c$ & $29 c$ & $43 d$ & $8,400 \mathrm{~b}$ & $9 b$ & $96 b$ \\
\hline \multirow[t]{2}{*}{ Media } & 17 & 27 & 5,000 & 15 & 161 & 37 & 57 & 7,775 & 11 & 129 \\
\hline & \multicolumn{5}{|c|}{ Ébano, San Luis Potosí P-V 2013 (temporal) } & \multicolumn{5}{|c|}{ Cotaxtla, Veracruz P-V 2014 (temporal) } \\
\hline RB-Pirulí & $32 \mathrm{~b}$ & $40 \mathrm{~b}$ & $9,300 \mathrm{a}$ & $15 a$ & $166 \mathrm{a}$ & $90 \mathrm{a}$ & $121 \mathrm{a}$ & $26,200 \mathrm{a}$ & $17 \mathrm{a}$ & $191 \mathrm{a}$ \\
\hline RB-Cañero & $28 \mathrm{c}$ & $35 c$ & $8,000 \mathrm{~b}$ & $10 \mathrm{~b}$ & $113 c$ & $45 b$ & $59 \mathrm{c}$ & $13,000 \mathrm{~b}$ & $16 a$ & $180 \mathrm{a}$ \\
\hline G-Star 501 & $37 \mathrm{a}$ & $45 a$ & $10,700 \mathrm{a}$ & $13 \mathrm{a}$ & $144 b$ & $49 \mathrm{~b}$ & $66 \mathrm{~b}$ & $14,200 \mathrm{~b}$ & $13 b$ & $153 b$ \\
\hline Media & 29 & 36 & 9,333 & 13 & 141 & 45 & 60 & 17,800 & 15 & 175 \\
\hline
\end{tabular}

oBrix: sólidos solubles; BTO: biomasa total; BTA: biomasa del tallo; ATJ: azúcares totales del jugo. Medias con letras iguales en las columnas dentro de cada localidad no son estadísticamente diferentes (Tukey, 0.05)

de semilla, el peso promedio de 1000 granos es de $18.9 \mathrm{~g}$, el tamaño de la semilla es mediana, de color marrón claro y sin latencia. La biomasa total se distribuye en $82 \%$ en los tallos, $12 \%$ en las hojas y $6 \%$ en la panoja. La superioridad promedio de RB-Pirulí sobre RB-Cañero fue de $40 \%$ para la biomasa de tallo, $33 \%$ para la biomasa total, $40 \%$ para el jugo del tallo (22\% en ${ }^{\circ}$ Brix y $9 \%$ en azúcares totales). Destaca el comportamiento de esta nueva variedad en la localidad de Cotaxtla, Veracruz en el ciclo P-V 2014 (Cuadro 1)
RB-Pirulí no es tolerante al pulgón amarillo [Melanaphis sacchari (Zehntner)]. En el centro de Veracruz ha mostrado tolerancia a roya (Puccinia purpurea Cooke), tizón foliar [Exserohilum turcicum (Pass.) K. J. Leonard \& Suggs] y antracnosis foliar [Colletotrichum graminicola (Ces.) G. W. Wilson]

En noviembre del 2017 se obtuvo el registro de esta variedad en el Catálogo Nacional de Variedades de Plantas con el número SOG-284-231117 y en julio del 2018 se

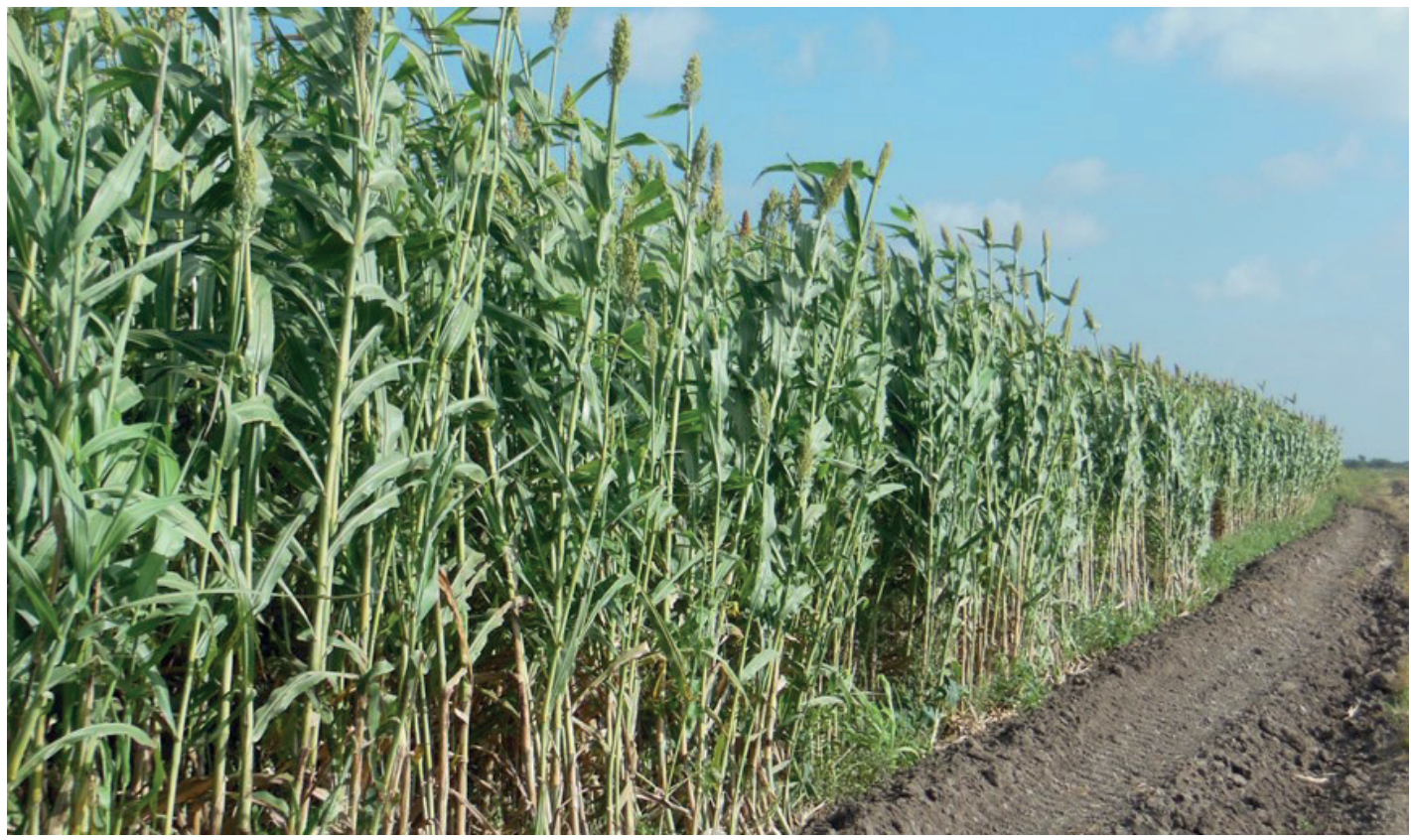

Figura 1. Variedad de sorgo dulce RB-Pirulí en etapa de floración. Rio Bravo, Tamaulipas, 0-I 2015. 
otorgó el Título de Obtentor No. 1890 por parte del Servicio Nacional de Inspección y Certificación de Semillas. La semilla se encuentra disponible en el Campo Experimental Rio Bravo, en Tamaulipas.

\section{AGRADECIMIENTOS}

Al Fondo Sectorial SAGARPA-CONACYT por el financiamiento al Proyecto 173411 "Mejoramiento genético de sorgo dulce para generar variedades con alto rendimiento agronómico y alto contenido de azúcares para la producción de bioetanol."

\section{BIBLIOGRAFÍA}

Barnaud A., G. Trigueros, D. McKey and H. I. Joly (2008) High outcrossing rates in fields with mixed sorghum landraces: how are landraces maintained? Heredity 101:445-452, https://doi.org/10.1038/ hdy.2008.77

Montes G. N., V. Pecina Q., M. E. Cisneros L. y M. A. García G. (2010) RB Cañero: sorgo dulce [Sorghum bicolor (L.) Moench] para la producción de etanol. Folleto Técnico No. 43. Campo Experimental Río Bravo. Instituto Nacional de Investigaciones Forestales, Agrícolas y Pecuarias. Río Bravo, Tamaulipas. $31 \mathrm{p}$

Murray S. C., W. L. Rooney, M. T. Hamblin, S. E. Mitchell and S. Kresovich (2009) Sweet sorghum genetic diversity and association mapping for brix and height. The Plant Genome 2:48-62, https://doi. org/10.3835/plantgenome2008.10.0011

Pedersen J. F., J. J. Toy and B. Johnson (1998) Natural outcrossing of sorghum and sudangrass in the Central Great Plains. Crop Science 38:937-939, https://doi.org/10.2135/cropsci1998.001 1183X003800040009x 
